\title{
Azione terapeutica dell'acqua oligominerale Sant'Elena di Chianciano nella calcolosi e nelle infezioni delle vie urinarie
}

\author{
N. Di Paolo, G. Garosi, S. Brardi, E. Gaggiotti, L. Capotondo \\ U.O. di Nefrologia e Dialisi, Azienda Ospedaliera Senese, Siena
}

a terapia idropinica rappresenta al momento attuale uno dei capisaldi fondamentali della terapia preventiva e curativa sia della calcolosi che delle infezioni delle vie urinarie (1-7). Le osservazioni mediche al riguardo datano da millenni, e la terapia idropinica è stata praticata da sempre sia in ambienti termali che nei presidi ospedalieri nonché al domicilio del paziente. Il concetto di potere terapeutico delle acque è insito nella natura umana, tanto è vero che, ancora oggi, popolazioni primitive sparse per il pianeta, mostrano tutte indistintamente la credenza ai poteri terapeutici di alcune acque sorgive. La medicina moderna si è occupata negli ultimi duecento anni, specie in Europa, del ruolo della terapia con acque minerali in queste affezioni delle vie urinarie, anche se ha sempre incontrato ed incontra tuttora difficoltà ad effettuare studi controllati che possano essere esenti da criticismi (810). Questi inconvenienti nascono dal fatto che fino ad alcuni anni or sono, la medicina termale in generale ed anche la terapia idropinica è stata sempre fondata sull'osservazione del malato e sulla modificazione del suo stato di benessere, e ci si è limitati ad ipotizzare vari mecca- nismi di azione di tale tipo di terapia. Oggi, quando tutto ciò non è più sufficiente, ma si esige una quantificazione scientifica del fenomeno, una sua valutazione statistica e una spiegazione razionale del meccanismo d'azione, lo sperimentatore si trova di fronte a problematiche complesse e di difficile soluzione (11).

Prima di tutto bisogna definire se l'acqua minerale bevuta alla fonte abbia la stessa azione di quella imbottigliata: è esperienza comune, specie nel campo della calcolosi, che pazienti con piccoli calcoli emettono nelle sedi termali calcoli e renella, mentre le stesse quantità della medesima acqua bevute a domicilio non provocano la stessa azione. Questo farebbe presupporre proprietà fisico-chimiche delle acque minerali ancora sconosciute.

In secondo luogo è ancora da definire il meccanismo d'azione della terapia idropinica sia nella prevenzione e trattamento della calcolosi che nelle infezioni delle vie urinarie: in particolar modo, a parte l'osservazione diretta dei risultati, mancano ancora sufficienti dati per poter definire quali siano gli indici urinari che possono aiutare a comprendere i fenome- ni che giustifichino questi risultati e siano significativi al riguardo.

Sono logicamente scontate le modificazioni di alcuni parametri quali l'osmolarità, la sodiemia, il pH ed altre legate alla diluizione urinaria dopo carico idrico e che sicuramente quantizzano il fenomeno del wash-out, capace di per sé di detergere le vie di deflusso; ma queste non sono sufficienti a spiegare l'intero fenomeno.

Negli ultimi anni, nel campo di ricerca della calcolosi urinaria, si sono sempre più andati affermando i ruoli della citraturia e della magnesuria e nel campo dell'infezione delle vie urinarie quelli delle interleuchine 6 ed 8 (1, 12-14). Il citrato, infatti, è un potente inibitore della formazione dei calcoli di ossalato di calcio e di fosfato di calcio (15) ed anche il magnesio urinario (16) ha sicuramente la capacità di inibire la formazione dei calcoli renali; spesso poi la calcolosi stessa è accompagnata da infezione delle vie urinarie e addirittura queste ultime possono favorire o causare la formazione di calcoli ed allora la terapia idropinica agisce su due direttive.

E nostro parere che sia indispensabile continuare a cercare ancora nuovi para- 
metri di monitoraggio proprio perché la sensibilità necessaria a quantificare una terapia cronica con acque minerali nella patologia calcolotica ed infettiva dell'apparato urinario deve essere necessariamente molto fine ed accurata.

L'Acqua oligominerale Sant'Elena di Chianciano è già nota da decenni per la sua attività terapeutica sulla calcolosi renale e sull'infezione delle vie urinarie (9, 17-24). L'ipotonia dell'acqua Sant'Elena esercita sul rene la tipica azione delle acque a bassa pressione osmotica attivando una diuresi proporzionale di solito alla quantità di acqua ingerita con un innalzamento del $\mathrm{pH}$ urinario. La presenza del magnesio, oltre alla azione antispastica e sedativa, assicura un importante fattore inibente la cristallizzazione.

Con lo scopo di meglio chiarire l'azione terapeutica di questa acqua oligominerale sulla calcolosi renale e sull'infezione delle vie urinarie, abbiamo intrapreso una sperimentazione clinica controllata tendente ad evidenziare l'effetto diretto dell'acqua su queste affezioni e a ricercare eventuali modificazioni della biochimica urinaria che possano aiutare a spiegare ulteriormente il meccanismo di azione.

\section{Materiali e Metodi}

Sono stati arruolati 47 pazienti afferenti agli Ambulatori dell'U.O. di Nefrologia e Dialisi dell'Azienda Ospedaliera di Siena ed all'Ambulatorio delle Terme Sant'Elena di Chianciano Terme. I pazienti erano 35 maschi e 12 femmine di età compresa tra i 16 ed i 75 anni con una media di $55.17 \pm 16.20$ per i maschi e di $46.66 \pm 18.86$ per le femmine. 23 soggetti erano affetti da calcolosi delle vie urinarie, 18 soggetti da infezione delle vie urinarie e 6 da calcolosi ed infezione contemporaneamente.

Sono stati esclusi dalla sperimentazione i soggetti affetti da affezioni sistemiche gravi, patologia prostatica occlusiva, cardiopatia scompensata, disturbi involutivi cerebrali, epatopatie croniche, terapie in atto con diuretici, alcalinizzanti, uricosurici, antibiotici e antiinfiammatori, sali minerali in generale.

La diagnosi di calcolosi delle vie urinarie è stata accertata mediante esami ecotomografici e radiologici, mentre l'infezione delle vie urinarie è stata accertata con esami delle urine ed urinocolture. A tutti i pazienti, previa ampia informazione dello scopo della sperimentazione e dopo aver ottenuto il consenso scritto, è stato somministrato a caso un quantitativo di due litri die di Acqua oligominerale Sant'Elena di Chianciano (a 25 soggetti) o due litri di acqua dell' acquedotto di Siena (a 22 soggetti).

L'acqua dell'acquedotto di Siena è stata scelta perché non si discosta molto nella sua composizione da quella della Sant'Elena di Chianciano Terme, perché è il risultato di una miscelazione delle acque provenienti dalle fonti del Vivo sull'Amiata e del Luco nel Chianti.

Le caratteristiche dell' Acqua oligominerale Sant'Elena di Chianciano Terme utilizzata per la sperimentazione sono le seguenti:

$\begin{array}{ll}\text { Residuo fisso a } 180^{\circ} & 473 \mathrm{mg} / \mathrm{lt} \\ \mathrm{Na}^{+} & 24.8 \\ \mathrm{~K}^{+} & 0.9 \\ \mathrm{Ca}^{++} & 123.7 \\ \mathrm{Mg}^{+} & 13.6 \\ \text { Durezza in }{ }^{\circ} \mathrm{F} & 36.5 \\ \mathrm{pH} & 7.3\end{array}$

La composizione dell'acqua dell'acquedotto di Siena utilizzata per la sperimentazione è la seguente:

$\begin{array}{ll}\text { Residuo fisso a } 180^{\circ} & 526 \mathrm{mg} / \mathrm{lt} \\ \mathrm{Na}^{+} & 36.8 \\ \mathrm{~K}^{+} & 2.1 \\ \mathrm{Ca}^{++} & 144 \\ \mathrm{Mg}^{+} & 2.2 \\ \text { Durezza in } & { }^{\circ} \mathrm{F} \\ \mathrm{pH} & 49.8 \\ & 7.34\end{array}$

La sperimentazione ha avuto la durata di 15 giorni per paziente. In condizioni basali e dopo 7 e 15 giorni di terapia, oltre alle note soggettive ed obbiettive inerenti le affezioni urinarie, sono stati valutati i seguenti parametri: ecotomografia renale, pressione arteriosa, peso corporeo, uricemia, calcemia, fosforemia, esame urine completo di sedimento urinario, il Cytur test urinario come determinazione aggiunta e semiquantitativa della leocucituria.

Nelle urine delle 24 ore sono stati determinati sempre ai medesimi tempi il $\mathrm{pH}$, l'osmolarità, il Calcio, il Sodio, il Potassio, il Magnesio, l'Acido urico, il Fosfato, il Citrato, l'N - acetil, B, D, glucosaminidasi (NAG), la B2-microglobulina, l'Alanino-aminopeptidasi (AAP) il Retinol Binding Protein (RBP), l' $\alpha$-1micro- globulina, la proteinuria l'interleuchina 6 e l'interleuchina 8.

Le metodiche attuate per la determinazione dei parametri urinari sopracitati sono state le seguenti:

pH urinario: Emogasometro Stat Profile 1 (Gepa)

Osmolarità urinaria: Osmometro di Fiske One-Ten (Sarin)

Calcio e Citrato: Spettrofotometro U-2000 Hitachi (kit BoehringerMannheim)

Ossalato: Spettrofotometro U-2000 Hitachi (kit SIGMA)

Retinol Binding Protein, $\alpha-1$-Microglobulina, B2-Microglobulina: Nefrolometro BNA 100 (kit Boehringer)

Magnesio, Ac. Urico e Fosforo: Colorimetro A U 5000 Olimpus (kit Merck)

NAG e AAP: Spettrofotometro U-2000

Hitachi (kit FAR)

Proteinuria: Metodo di Esbach

Interleukina 6: Elisa, Eurogenetics

Interleukina 8: Elisa, Tecnogenetics.

E stata inoltre annotata la palatabilità dell'acqua, la tolleranza gastrica e qualsiasi evento indesiderato.

I dati ottenuti sono stati processati con test non parametrici per dati appaiati (Wilcoxon Test).

\section{Risultati}

L'Acqua oligominerale Sant'Elena di Chianciano produce un aumento giornaliero medio della diuresi con una diminuzione dell'osmolarità urinaria. È una proprietà delle acque oligominerali "veri diuretici fisiologici" che consente di eliminare un volume di urina superiore al volume di acqua ingerito e provoca un aumento dell'escrezione di urina, generalmente nel giro di tre ore. Il volume di urina eliminato in sovrappiù si aggira intorno ai $300 \mathrm{~g}$ per un litro di acqua assunto. Anche nei controlli si è avuto un risultato similare, ma leggermente inferiore. Le differenze sostanziali tra i pazienti afferenti alle Terme Sant'Elena di Chianciano e quelli trattati con terapia idropinica con acqua dell'acquedotto di Siena sono state le seguenti:

- Nessun paziente ha dichiarato intolleranza gastrica od effetti collaterali con l'acqua Sant'Elena, mentre 6 pazienti di controllo hanno riferito intolleranza gastrica (in tre casi), pirosi 
(in due casi) e gastralgia (in un caso).

- Tutti i pazienti trattati con Acqua Sant'Elena si sono dichiarati molto soddisfatti della palatabilità dell'acqua, mentre solo 7 di quelli di controllo hanno dichiarato buona l'acqua dell'acquedotto di Siena.

- In 6 pazienti afferenti alle Terme Sant'Elena di Chianciano si sono avute emissioni di renella (in cinque casi) e di un calcolo (in un caso). Nessuno dei pazienti di controllo ha emesso renella o calcoli durante la sperimentazione.

- La citraturia (Fig. 1) e la magnesiuria (Fig. 2) hanno subito un incremento, dopo terapia idropinica, mediamente più elevato nei pazienti trattati con Acqua Sant'Elena rispetto a quelli trattati con acqua dell'acquedotto. Per quanto riguarda la citraturia la differenza tra il basale ed il $14^{\circ}$ giorno tra i pazienti delle Terme Sant'Elena e i controlli è risultata significativa $(\mathrm{p}<0.05)$.

- Nei pazienti calcolotici non si sono notate modificazioni delle interleuchine urinarie 6 ed 8 . Estrapolando solo i pazienti con infezione delle vie urinarie (nel gruppo di quelli trattati con Acqua Sant'Elena e di quelli trattati con acqua dell'acquedotto di Siena), la terapia idropinica ha fatto diminuire, dopo 15 giorni di terapia, i valori di interleuchina 6 nei pazienti trattati con Acqua Sant'Elena rispetto ai valori basali; la diminuzione dell'interleuchina 6 nei pazienti trattati con Acqua oligominerale Sant'Elena è risultata statisticamente significativa (Fig. 3).

- Sempre nei pazienti con infezioni delle vie urinarie trattati con Acqua Sant'Elena, si è notata una diminuzione evidente degli enzimi urinari NAG e LAMP, mentre tale fenomeno non si è osservato nei soggetti trattati con acqua dell'acquedotto di Siena. I risultati riscontrati hanno determinato nel giro di due settimane un decremento degli enzimi AAP, NAG, LAP, del Lisozima e della proteinuria, significativi attestati di attività antiflogistiche reali, protettive dell'epitelio renale e tubulare nelle infezioni urinarie.

- In tutte e due le categorie dei pazienti con infezione delle vie urinarie si è dimostrata una diminuzione non si-

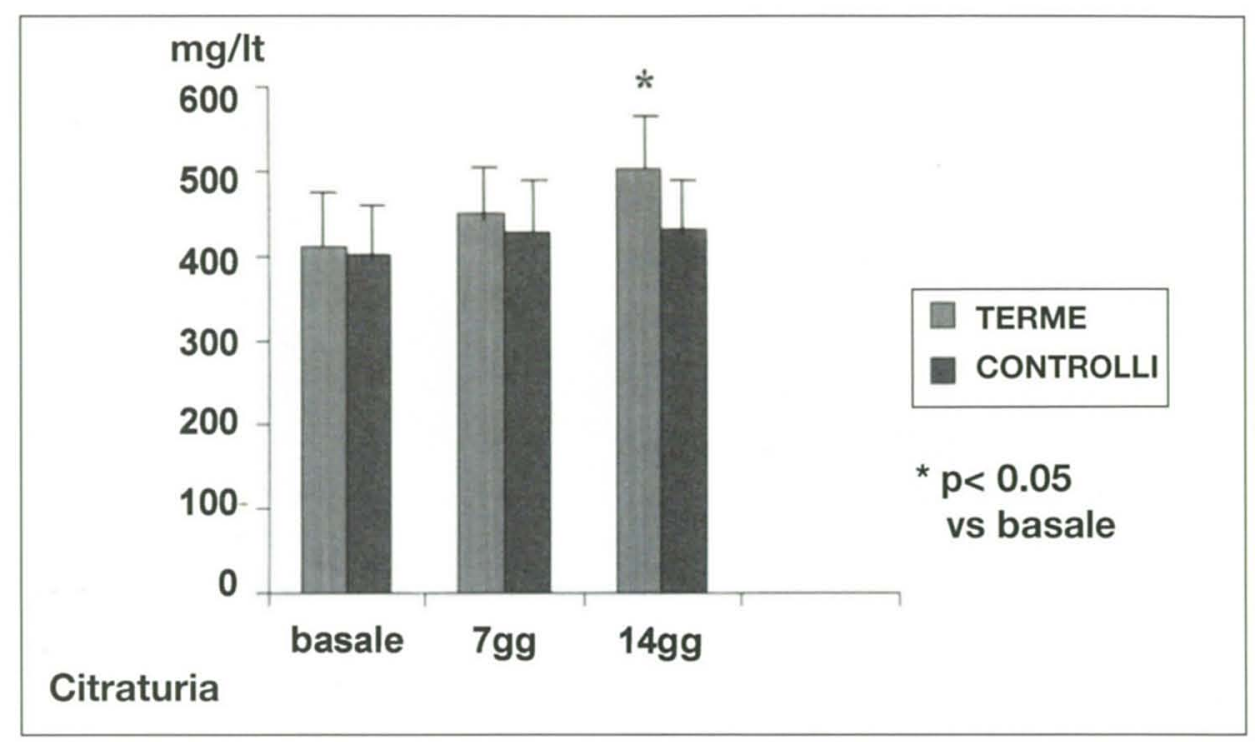

Fig. 1 - Livelli di citrato urinario o pari tempi nei pazienti trattati con Acqua Sant'Elena e nei controlli.

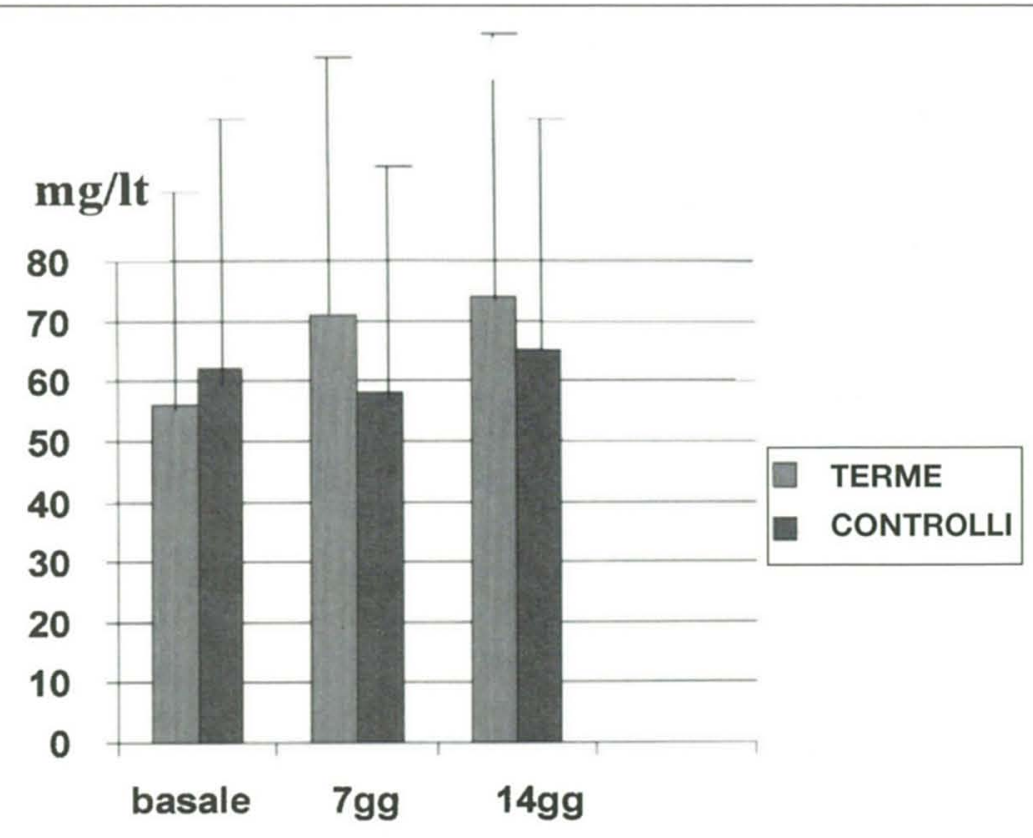

Magnesiuria

Fig. 2 - Livelli di magnesio urinario a pari tempi nei pazienti trattati con Acqua Sant'Elena e nei controlli.

gnificativa della leucocituria (Fig. $4)$, fin dal $7^{\circ}$ giorno $(\mathrm{p}<0.05)$, mentre si accentua al $14^{\circ}$ giorno di terapia $(\mathrm{p}<0.01)$.

- Nessuno degli altri parametri esaminati (calciuria, ossaluria, rbp, fosfaturia), ha dimostrato modificazioni fra i valori basali e dopo terapia idropinica a parte l'aumento modesto del pH e dell'uricuria.

\section{Discussione}

Fin dall'antichità è noto che l'aumentata diuresi riduce il rischio della formazione dei calcoli renali. Molto più recentemente Pak et al $(25,26)$ nel 1980 e nel 1981 hanno dimostrato che la diluizione urinaria riduce la cristallizzazione dei sali di calcio, fattore importante se si pensa che 


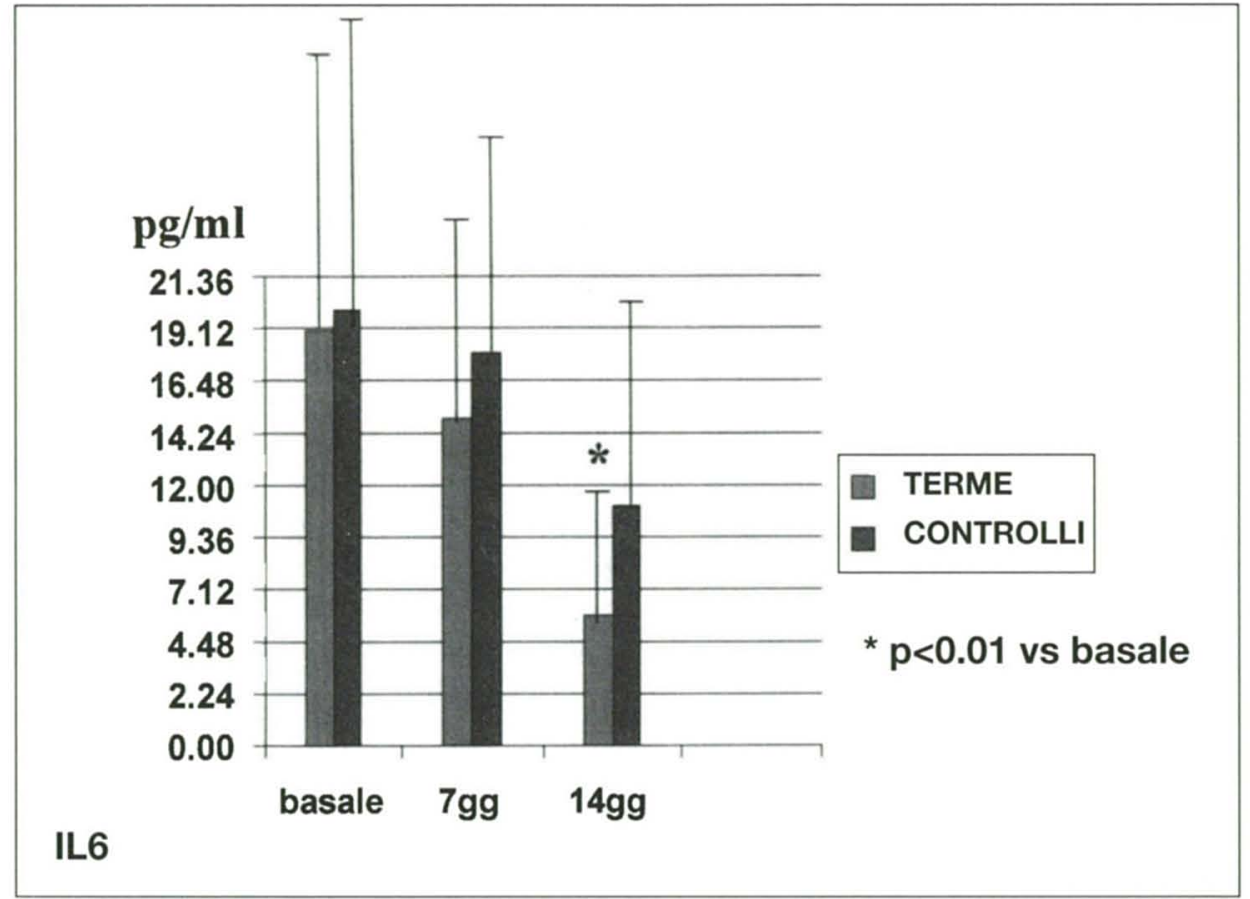

Fig. 3 - Livelli urinari di Interleukina 6 a pari tempi nei pazienti trattati con Acqua Sant'Elena e nei controlli.

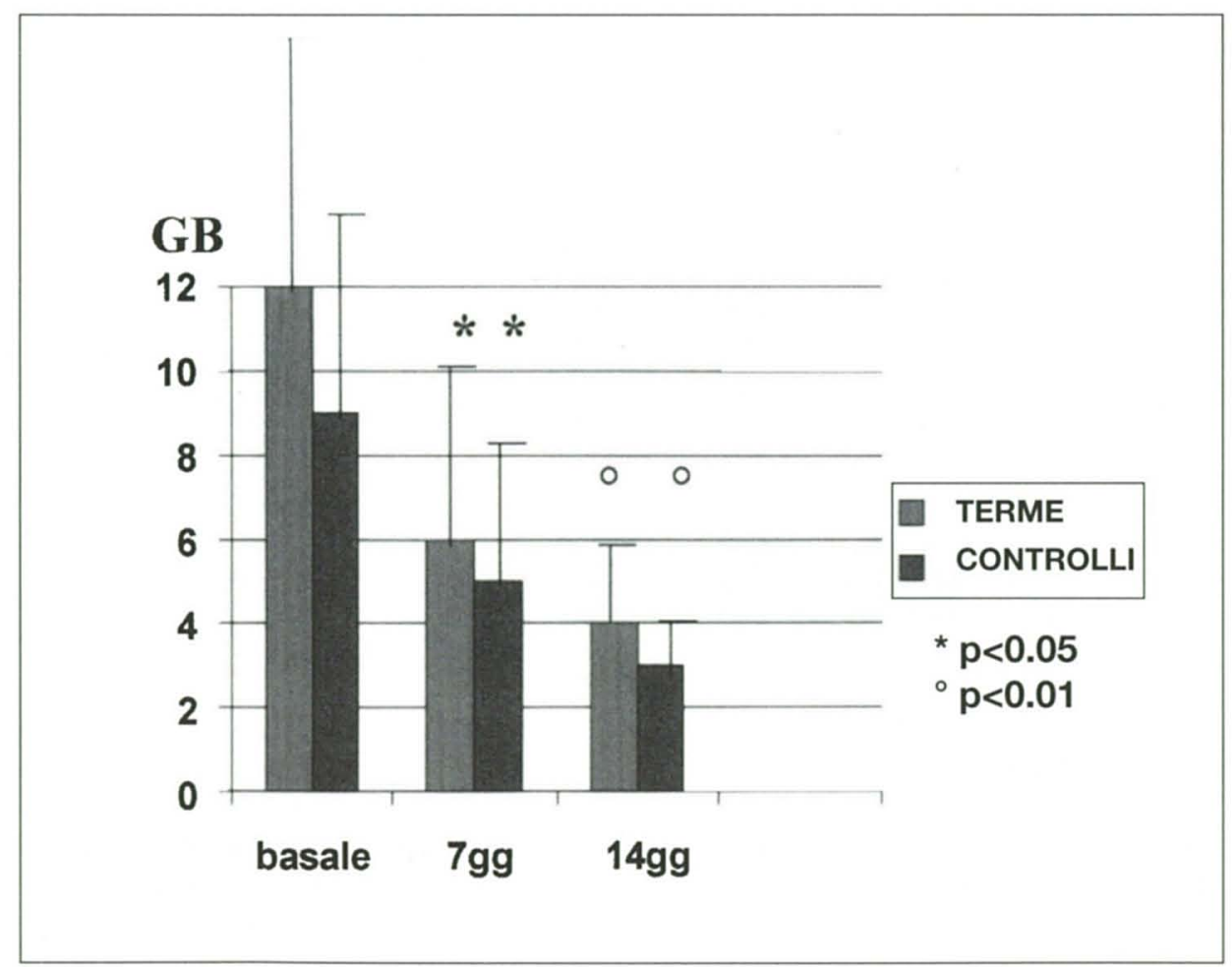

Fig. 4 - Leucociti urinari osservati per campo a 400 x a pari tempi nei pazienti trattati con Acqua Sant'Elena e nei controlli.

Sriboonlue et al (27) hanno potuto dimostrare che nei soggetti affetti da calcolosi renale la supersaturazione urinaria e la cristallizzazione sono più frequenti nei soggetti calcolotici che nei normali, perfino in urine a basso peso specifico, ed alle stesse conclusioni sono arrivati Marangella et al (28) nel 1985.

Aroldi et al (29) nel 1985 hanno evidenziato che l'elevato apporto idrico, associato alla dieta, può prevenire la formazione di calcoli renali in pazienti senza ipercalciuria ed iperuricuria, anche se nei pazienti ipercalciurici ed ipericosurici l'associazione con allopurinolo da esito ai migliori risultati. Anche Jaeger (5) nel 1994 ha indicato la terapia idropinica e la dieta quali primi presidi da utilizzarsi in pazienti affetti da calcolosi calcica. Alle stesse conclusioni è arrivato Ackermann (30) nel 1990, aggiungendo che l'apporto calcico deve essere, in assenza di ipercalciuria, nell'adulto di circa 1 grammo/die, mentre basso deve essere l'apporto di sodio.

Il problema dell'apporto calcico nella dieta del paziente affetto da calcolosi renale calcica ci sembra ormai ben chiarito, specialmente nel paziente affetto da ipercalciuria idiopatica nel quale l'assorbimento intestinale di ossalato è aumentato se si riduce la concentrazione di calcio intestinale: infatti normalmente l'assorbimento intestinale di $\mathrm{Ca}$ è molto basso $(5-10 \%)$ in quanto viene precipitato dal calcio nell'ambiente alcalino intestinale: in caso di scarso apporto di calcio o di aumento del suo assorbimento intestinale, anche l'assorbimento di ossalato è aumentato.

Quindi l'aumento della calcemia tende a ridurre il calcio intestinale con tendenza all'assorbimento di ossalato che a sua volta produce iperossaluria che avrà luogo solo se esiste un intake di ossalato sufficiente (31). In sintesi se ne può dedurre che acque minerali contenenti grandi quantità di calcio sono controindicate nella calcolosi renale, ma che modeste quantità di calcio, come sono presenti nell'Acqua Sant'Elena, possono definirsi ideali nella composizione di un'acqua minerale da utilizzarsi nella terapia idropinica della calcolosi renale (32).

Alcuni oligoelementi come il calcio e in misura minore il magnesio aumenterebbero la permeabilità cellulare; a livello delle strutture lipoproteiche della membrana cellulare, il calcio e il magnesio andrebbero infatti a sostituirsi agli ioni monovalenti $\mathrm{Na}^{+}$e $\mathrm{K}^{+}$; in questo lo ione $\mathrm{Ca}^{++}$neutralizza le forze elettrostatiche che mantengono in posizione chiusa $\mathrm{i}$ pori cellulari; ottenuta in questo modo l'apertura dei pori, l'acqua oligominerale può permeare la cellula e, in virtù della sua ipotonicità, esplicare non solo il suo effetto di sottrazione di liquidi ma anche di scorie metaboliche attuandosi così una vera e propria azione di "lavaggio cellulare" $(33,34)$. Ackermann et al (30) affermano addirittura che l'ingestione di 
acqua ad alto contenuto calcico aumenta la calciuria, ma diminuisce l'ossaluria in contrasto con l'acqua minerale povera di calcio.

Rodgers (35) nel 1997 ha osservato come le acque minerali contenenti magnesio e modeste quantità di calcio devono considerarsi come un agente terapeutico o profilattico della calcolosi renale da ossalato di calcio.

Borghi et al (3) nel 1996 in uno studio controllato su 199 pazienti affetti da calcolosi renale calcica condotto per 5 anni, hanno potuto dimostrare che due litri di acqua al dì sono da considerarsi la prima terapia da effettuarsi in pazienti affetti da calcolosi renale, mentre nei pazienti con ipercalciuria dovrebbe essere prescritta anche una dieta specifica ed una appropriata terapia farmacologica.

D'altra parte molti Autori $(16,36)$ hanno evidenziato il ruolo di sostanze urinarie inibitrici la cristallizzazione e di conseguenza la formazione di calcoli nelle vie urinarie. Fra queste sostanze, il citrato ed il magnesio sembrano fra le più importanti (15, 37-40).

Per quanto riguarda l'infezione delle vie urinarie è ben nota l'azione terapeutica del wash-out $(2,41)$ ed oggi possiamo tranquillamente affermare di avere a disposizione parametri di valutazione quanto mai significativi nell'evidenziare anche modeste alterazioni flogistiche. Naturalmente la leucocituria e la batteriuria, questa ultima evidenziata con esami colturali, sono le due più importanti manifestazioni dell'infezione urinaria, ma le determinazioni degli enzimi urinari possono darci informazioni sull'ubicazione del danno renale, mentre la più recente introduzione della determinazione delle interleuchine 6 ed 8 ci permette di intercettare anche modeste alterazioni flogistiche non detettabili con gli altri parametri più facilmente disponibili (1214, 42).

Nella presente sperimentazione tali acque sono state somministrate per os alla dose di due litri/die in individui affetti da calcolosi delle vie urinarie e/o da infezione delle vie urinarie. Come era facile prevedere, la palatabilità dell' Acqua Sant'Elena è decisamente migliore di quella di un'acqua proveniente da un acquedotto comunale, che è clorata, ma è da sottolineare il fatto che due litri di acqua al dì sono perfettamente sopportati dai pazienti che bevono la Sant'Elena, mentre ci sono quasi il $30 \%$ dei soggetti

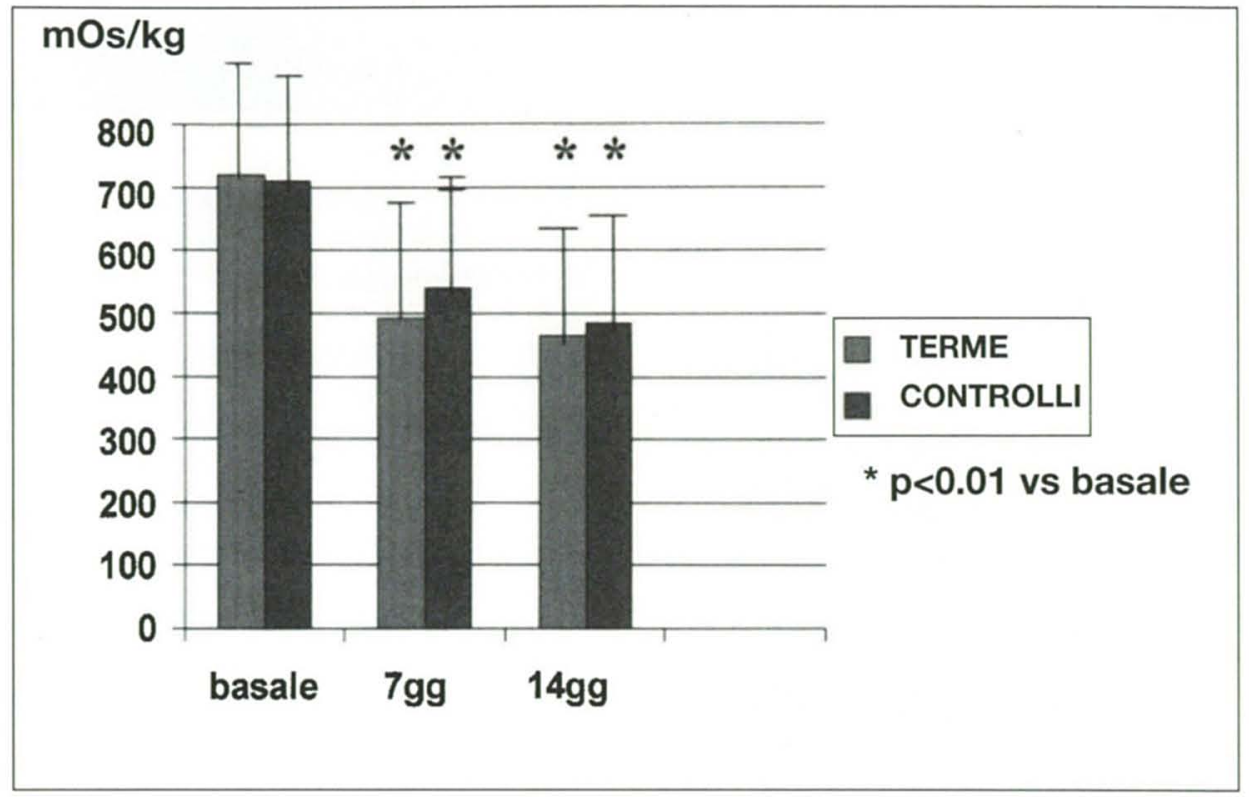

Fig. 5 - Valori di Osmolarità urinaria a pari tempi nei pazienti trattati con Acqua Sant'Elena e nei controlli.

che bevono due litri dell'altra acqua che accusano una serie di fastidi.

Per quanto riguarda i pazienti calcolotici, questi sono riusciti in oltre il $20 \%$ dei casi ad espellere renella o calcoli con terapia idropinica con acqua Sant'Elena, mentre tale effetto non si è registrato nei pazienti che hanno bevuto acqua dell'acquedotto. Questo fenomeno in verità è frequente nella terapia termale e non ha trovato, fino ad oggi, una convincente spiegazione scientifica razionale. Non si sono avute modificazioni rilevanti a livello del $\mathrm{pH}$ urinario, della calciuria e della ossaluria, della fosfaturia e dell'uricuria.

Fra i dati di laboratorio per i pazienti calcolotici emerge la proprietà dell' Acqua oligominerale Sant'Elena a mantenere livelli urinari elevati di citrato e magnesio, che, come abbiamo ricordato, sono da considerarsi elementi inibitori della cristallizzazione (Figg. 1, 2).

A nostro parere la significatività statistica per quanto riguarda la citraturia al $14^{\circ}$ giorno della sperimentazione è quanto mai importante: infatti dimostra che l'Acqua oligominerale Sant'Elena, rispetto all'acqua dell' acquedotto di Siena, di simile composizione, ha un effetto più marcato sulla citraturia che, come fattore inibente la formazione dei calcoli urinari, gioca un ruolo fondamentale nella prevenzione di questa malattia.

Anche la magnesuria aumenta con la terapia idropinica, più marcatamente nei soggetti trattati con Acqua Sant'Elena: ciò è abbastanza logico in quanto il contenuto di magnesio dell'Acqua Sant'Elena è molto più elevato di quello dell'acqua dell'acquedotto di Siena.

La calciuria e l'ossaluria non hanno mostrato differenze significative né prima né dopo trattamento, né fra i due gruppi di soggetti. Il risultato è abbastanza conforme a quanto rilevato da altri Autori in questo tipo di sperimentazioni $(43,44)$.

La diminuzione dei valori di citochine urinarie nei pazienti con infezione delle vie urinarie indica chiaramente che la terapia idropinica riesce a ridurre lo stato infiammatorio e tale fenomeno antiflogistico è più evidente con l'acqua Sant'Elena.

L'importanza diagnostica che vanno man mano assumendo le citochine urinarie nell'ambito delle affezioni renali infettive porta questa significatività ad assumere un ruolo importante per comprendere la capacità che ha l'Acqua oligominerale Sant'Elena di incidere sulla malattia infettiva renale, sia nel senso terapeutico favorendo la terapia antimicrobica, sia sull'effetto preventivo, essendo questo ultimo sicuramente l'effetto curativo più importante da perseguire con un' acqua minerale.

Per quanto riguarda la diminuzione della leucocituria (Fig. 4) e dell'osmolarità urinaria (Fig. 5), con valori significativi rispetto a quelli basali sia per l'Acqua oligominerale Sant'Elena che per quella dell' acquedotto, è evidentemente imputabile alla diluizione delle urine relativa 
al carico idrico, di per sé terapeutica, ma ovviamente non specifica.

La terapia idropinica di per sé è, come del resto era già noto, capace di influire positivamente sulla terapia e sulla prevenzione della calcolosi renale e sulle infezioni delle vie urinarie.

Oltre ai meccanismi d'azione già ben conosciuti, che sono riferibili alla aumentata diuresi e quindi all'effetto wash-out ed alla diminuzione dell'osmolarità urinaria, fattori questi che giocano ruoli terapeutici nella calcolosi e nell'infezione urinaria, emerge da questo studio sperimentale che l'Acqua oligominerale Sant'Elena di Chianciano è più attiva terapeuticamente di un'acqua d'acquedotto comunale e che tale riscontro è il risultato di particolari indagini urinarie eseguite utilizzando alcuni markers che hanno confermato tale asserzione.

\section{BIBLIOGRAFIA}

1. Andriolo VT. Water, acidosis and experimental pyelonephritis. J Clin Invest 1970; 49: 21-30.

2. Andriolo VT. Effect of water diuresis in chronic pyelonephritis. J Lab Clin Med 1968; 72: 1-16.

3. Borghi L, Meschi T, Amato F, Briganti A, Novarini A, Giannini A. Urinary volume, water and recurrences in idiopathic calcium nephrolithiasis: a 5-year randomized prospective study. J Urol 1996; 155: 839-43.

4. Gutenbrunner C, Gilsdorf K, Hildebarndt G. The effect of mineral water containing calcium on supersaturation of urine with calcium oxalate. Urologe 1989; 28 : 15-9.

5. Jaeger P. Prevention of recurrent calcium stones: diet versus drags. Miner Electrolyte Metab 1994; 20: 410-3.

6. Ljunghall S, Fellstrom B, Johansson G. Prevention of renal stones by a high fluid intake. Eur Urol 1988; 14: 381-5.

7. Marangella M, Vitale C, Petrarulo M, Rovera L, Dutto F. Effects of mineral composition of drinking water on risk for stone formation and bone metabolism in idiopathic calcium nephrolithiasis. Clin Sci 1996; 91: 313-8.

8. Di Paolo N. Semeiotica del rene e delle vie urinarie. Wichtig Ed. Milano 1995; 80-1.

9. Di Paolo N. L'acqua Sant'Elena di Chianciano e medicina termale nefrologica. Ed Lischi e Nistri, 1983 Pisa 1-95.

10. Hesse A, Siener R, Heynck $\mathrm{H}$, Jahnen A. The influence of dietary factors on the risk of urinary stone formation. Scanning Microsc 1993; 7: 1119-27.
11. Vanherweghem JL, Quenon M, Dhaene M, et al. Comparative effects of oral water load of different mineral composition on the excretion of ions and oxalic acid. Nephrologie 1984; 5: 184-8.

12. Agace WW, Hedges SR, Ceska M, Svamborg C. Inerleukin8 and neutrophil response to mucosal gram-negative infection. J Clin Invest 1993; 92: 780-5.

13. Benson M, Jodal U, Agage $\mathrm{W}$, et al. Interleukin 6 and IL 8 in children with febrile urinary tract infection ans asymptomatic bacteriuria. J Infect Dis 1996: 174: 1080-1.

14. Mukaida N, Ishiyama S, Tokue A, Kawai T, Matsushima $\mathrm{K}$, Kasahara T. Infect Immun 1993; 61: 1307-11.

15. Goldberg H, Gass L, Vogl R, Rapaport A, Oreopoulos DG. Urine citrate and renal stone disease. Canad Med Ass J 1989; 141: 217 21.

16. Trinchieri A, Mandreassi A, Luongo P, Rovera F, Longo G. Urinary excretion of citrate, glycosaminoglycans, magnesium and zinc in relation to age and sex in normal subjects and in patients who form calcium stones.

17. Pietravalle N. Azione biologica e terapeutica dell'acqua Sant'Elena di Chianciano. Ed Tip Madonna delle Querce, Chianciano, 1938.

18. Spadea G, Serra F, Schiano G. L'acqua Sant'Elena nella cura della nefolitiasi. Proceedings of Internal Soc of Med Hydrol 1950; 3-8.

19. Bocconi G, Franceschetti L, Serra F. Sulla prova di cristalluria provocata nello studio dell'azione diuretica di un'acqua minerale. Minerva Medica 1955; 2: 1-4.

20. Bocconi G. Ancora sull'azione antispastica delle acque minerali nella nefrolitiasi. Clinica Termale 1953; 6: 1-5. 
21. Bocconi G. L'azione di un' acqua minerale nelle cistite sperimentale. Clinica Temale 1956; 4: 44-7.

22. Bocconi G. L'acqua Sant'Elena di Chianciano. Ed. Tipografia Nuova, Chianciano 1964.

23. Di Paolo N, Sodi A, Buoncristiani U, et al. Sperimentazione policentrica sull'efficacia terapeutica dell'acqua Sant'Elena in alcune malattie delle vie urinarie. Atti del $48^{\circ}$ Congresso Nazionale dell'Associazione Medica Italiana di Idroclimatologia, Talassologia e Terapia Fisica, 1983; 153.

24. Vercellino G. L'azione dell'acqua Sant'Elena di Chianciano nell'infiammazione cronica della vescica urinaria (atti XXXIX Congr Naz Idrocl - Talass e Ter - Fis $4,1965)$.

25. Pak CY, Peters P, Hurt G, et al. Is selective therapy of recurrent nephrolithiasis possible? Amer J Med 1981; 71: 615-22.

26. Pak CY, Sakhaee K, Crowther C, Brinkley L. Evidence justifying a high fluid intake in treatment of nephrolithiasis. Ann Intern Med 1980; 93: 36-9.

27. Sriboonlue P, Prasongwattana V, Sriboonlue $M$, Chata $K$, Tunsanga K, Sitprija V. Low specific gravity urine with crystalluria as discriminant index for nephrolithiasis. J Med Ass Thai 1990; 73: 634-40.

28. Marangella M, Daniele PG, Ronzani M, Sonego S, Linari F. Urine saturation with calcium salts in normal subjects and idiopathic calcium stone formers estimated by an improved computer model system. Urol Res 1985; 13: 18993.

29. Aroldi A, Graziani G, Passerini $\mathrm{P}$, et al. High fluid intake or pharmacological therapy in recurrent stone former patients? Proc Eur Dial Tras Ass Eur Re Ass 1985; 21: 743-6.
30. Ackermann D. Prophylaxis in idiopathic calcium urolithiasis. Urol Res 1990; 18 (Supp 1): S3740.

31. Robertson WG, Latif AB, Scurr DS, Caswell AM, Drach GW, Randolph AD. Inhibitors of calcium oxalate crystallisation in urine stone formers and normal. In: Ryall $\mathrm{R}$ et al. Urinary Stone, Churchill Livingstone Ed, Melbourne, 1984; 167-72.

32. Robertson WG. Aetiological factors in stone formation. In: Cameron $\mathrm{S}$ et al. Exford Textbook of Clinical Nephrology, Oxford Ed, Oxford 1992; Vol III 1822-46.

33. Cristofolini M, Tommasi G. Indagine sulle variazioni della proteina C reattiva (P.C.R) di soggetti eczematosi dopo terapia balneotermale a Comano. Atti $\mathrm{X}^{\circ}$ Congr Naz A.D.O.I (Ass Dermat Osp It), L'Aquila, 1970.

34. Lenti G, Emanuelli G. L'enzimologia reno-urinaria, approcci istogenetici e clinici. Quad Sclavo Diagn 1973; 9: 417.

35. Rodgers AL. Effect of mineral water containing calcium and magnesium on calcium oxalate urolithiasis risk factors. Urol Intern 1997; 58: 93-9.

36. Rodman D, Kutner $\mathrm{MH}$, Redd SC, Waters WC, Gerron GG, Blejer J. Hypocitraturia in calcium nephrolithiasis. J Clin Endocrinol Metab 1978; 55: 1052-7.

37. Hosting DH, Wilson JW, Liedtke RR, Smith LH, Wilson DM. Urinary Citrate excretion in normal persons and patients with idiopathic calcium urolithiasis. J Lab Clin Med 1985; 106: 682-9.

38. Zerwekl JE, Hwang TI, Poindexter J, Hill K, Wendell G, Pak CY. Modulation by calcium of the inhibitory activity of naturally occurring urinary inhibitors. Kidney Inter 1988; 33: 1005-8.

39. Cowley DM, Whinney BC, Brown
JM, Chalmers AH. Effect of citrate on the urinary excretion of calcium and oxalate: relevance to calcium oxalate nefrolithiasis. Clin Chem 1989; 35: 23-8.

40. Nikkila M, Koivula T, Jokela H. Urinary citrate excretion in patients with urolithiasis and normal subjects. Eur Urol 1989; 16: 382-5.

41. Borgatti M, Malaguzzi PA. Ricerche microbiologiche sulla influenza di un'apporto idrico forzato con un' acqua bicarbonato-calcica sulla colonizzazione renale da Streptococcus faecalis. Min Idrologica 1971; 11: 53-6.

42. Hodgers $\mathrm{S}$, Anderson $\mathrm{P}, \mathrm{Li}$ din-Janson G, de Man P, Svanborg C. Interleukin 6 response to deliberate colonisation of the human urinary tract with gram-negative bacteria. Infect Immunol 1991; 59: 121-7.

43. Ackermann D, Baumann JM, Futterlieb A, Zingg EJ. Influence of calcium content in mineral water on chemistry and crystallization conditions in urine of calcium stone formers. Eur Urol 1988; 14: 305-8.

44. Baggio B, Gambaro G, Favaro $S$, et al. Juvenile renal stone disease: a study of urinary promoting and inhibiting factors. J Urol 1983; 130: 1133-5. 